\title{
An experimental investigation of tool nose radius and machining parameters on TI-6AL-4V (ELI) using grey relational analysis, regression and ANN models
}

\author{
Darshit R. Shah ${ }^{a^{*}}$ and Sanket N. Bhavsar ${ }^{\mathrm{b}}$
}

${ }^{a}$ Mechanical Engineering Department, L.D.College of Engineering, Ahmedabad, Gujarat, India ${ }^{b}$ G.H.Patel College of Engineering and Technology, (affiliated to GTU) Vallabh Vidyanagar, Gujarat, India

CH R O N I C L E

\begin{tabular}{l}
\hline Article history: \\
Received: October 28, 2018 \\
Received in revised format: De- \\
cember 25, 2018 \\
Accepted: January 10, 2019 \\
Available online: \\
January 10, 2019 \\
\hline Keywords: \\
Titanium Alloys \\
Grey Relational Analysis \\
Regression \\
Artificial Neural Network \\
ANOVA \\
Machining \\
Turning \\
Cutting force \\
Cutting temperature \\
Tool nose radius
\end{tabular}
\begin{abstract}
A B S T R A C T
Ti-6Al-4V Extra Low Interstitial (ELI) exhibits superior properties because of controlled interstitial element of iron and oxygen. The effects of four cutting parameters namely cutting speed, feed, depth of cut and tool nose radius on responses like cutting force, average cutting temperature and surface roughness have been investigated for turning of Ti-6Al-4V (ELI). Total 81 experiments have been performed in dry environment. Grey Relational Analysis has been used for multi-objective optimization. Analysis of Variance test has been carried out to investigate contribution of input parameters. The model was found fit with R-Square value of $88.74 \%$. Regression and ANN models are developed for prediction and compared. From the Grey relational analysis, it is clear that optimum parameters to minimize cutting force, cutting temperature and surface roughness while turning Ti-6Al-4V (ELI), are cutting speed as $140 \mathrm{rpm}$, Nose radius $1.2 \mathrm{~mm}$, Feed $0.051 \mathrm{~mm} / \mathrm{rev}$ and depth of cut is $0.5 \mathrm{~mm}$. In comparison of regression model, the ANN model is found to be more accurate with average error of $3.57 \%$.
\end{abstract}

\section{Introduction}

Superior and favorable mechanical properties have made titanium alloys, a perfect choice in the applications of aerospace, biomedical and marine applications. High strength to weight ratio, better corrosion resistance, and good fracture toughness are attractive properties possessed by titanium alloys. Despite having complimentary properties, titanium alloys fall under the category of difficult to cut materials because of poor thermal conductivity and rapid tool wear. The high cutting temperature is an issue which requires high attention as it is responsible for poor machinability (Narutaki et al., 1983). Ti-6Al-4V and Ti-6Al-4V ELI (Extra Low Interstitial) are basically developed to be used as structural material but it has found wide application as implant material too (Niinomi, 1998). The extra low interstitial (ELI) grade of

* Corresponding author. Tel.: +919925237030

E-mail address: darshit@ldce.ac.in (D. R. Shah) 
Ti-6Al-4V exhibits higher ductility and improved fracture stiffness than grade5 Ti-6Al-4V. This is because of controlled interstitial element of iron and oxygen. The investigation on diffusion bonding of Ti6Al-4V ELI was also carried out, which revealed that it is possible to have super-plastic forming and diffusion boding at lower temperature than conventional Ti-6Al-4V (Lee et al., 2007). The components to be used in aerospace field are expected to have better surface integrity and higher reliability. The investigation by Che-Haron and Jawaid (2005) revealed that the surface integrity is more affected by feed and tool nose radius while machining Ti-6Al-4V ELI. In order to understand fatigue behavior of implant, the investigation on the relation between fatigue damage and mechanical properties of Ti-6Al4V ELI was carried out by Akahori and Niinomi (1998). To evaluate the oxygen effect on processing of Ti-6Al-4V, the shapes of stress-strain curves, the kinetic parameters, and the processing maps obtained and have been compared for two grades of material (Prasad et al., 2001). Titanium alloys are used as implant materials for bio medical and dental application because of their corrosion resistance and good bio-compatibility. The corrosion behavior of titanium alloys like Ti-6Al-4V ELI and Ti-6Al-7Nb in simulated body fluids have also been investigated (Tamilselvi et al., 2006).

Turning is highly significant manufacturing process, in which single point cutting tool removes material from cylindrical work-piece while it is rotated. There are three cutting forces produced during turning namely thrust force, which acts in direction of cutting speed, feed force in the direction of feed and radial force which is produced in the direction normal to cutting speed. Effect of parameters on cutting power has been investigated by researchers (Valera \& Bhavsar, 2014). Many researchers have contributed their work on optimization of process parameters in order to improve machinability of titanium alloys. Significance of cutting parameters on Tool life and surface roughness of Ti-6Al-4V ELI was investigated (Sulaiman et al., 2013). The findings show that feed rate and cutting speed were highly influencing factors for surface roughness. Tool nose radius also affects the surface properties of the product (Yildiz, Irez, \& Sur, 2016). It has been observed that cutting speed and feed have more influence on cutting temperature (Nath et al., 2017). The geometry of cutting tool is also significant. Xie et al. (2013) investigated the effect of micro-grooved tool on cutting temperature and cutting force while dry turning of titanium alloy, and reported the decrease in cutting temperature with decrease in micro groove depth. After prolonged machining of titanium alloy under dry environment, tearing and plastic deformation of machined surface were observed (Che-Haron \& Jawaid, 2005). To improve tool life during machining of titanium alloy, use of solid lubrication is a better option as it can perform cooling and lubrication simultaneously (Moura et al., 2015). Investigation of effect of cutting speed, feed and depth of cut on cutting temperature while turning hardened steel EN-36 was carried out by researchers(Gosai \& Bhavsar, 2016). Grey Relational Analysis (GRA) is an effective tool for multi objective optimization. Many researcher have used the GRA method for optimization of parameters (Maiyar et al., 2013; Sarıkaya \& Güllü, 2015; Vinayagamoorthy \& Anthony Xavior, 2014). It has been effectively used for optimization of thermally enhanced machining parameters while turning Inconel 718 (Ganta et al., 2017). Optimization of cutter geometric parameters while end milling of titanium alloy was also carried out (Ren et al., 2015). Investigation of drilling parameters on hybrid polymer composite revels the important significance of parameters on delamination, thrust force and torque (Anand et al., 2018). In recent times artificial intelligence has drawn attention of many researchers. Amongst various methods based on artificial intelligence, Artificial Neural Network has been widely used by many researchers to predict the responses. The prediction of surface roughness has been predicted using ANN model and multiple regression method by Asiltürk and Çunkaş (2011). They concluded that ANN model is powerful tool for prediction as compared to multiple regression model. Machining of AISI 1030 steel by PVD and CVD coated tool by varying feed rate and cutting speed has been investigated, and the surface roughness was predicted by ANN model with acceptable accuracy (Nalbant et al., 2009). As per the literature survey, very limited research work has been carried out on simultaneous effect of cutting parameters and tool geometry on surface roughness, cutting temperature and cutting force while turning Ti-6Al-4V (ELI). In this study, an attempt has been made to investigate the effect of cutting speed, feed, depth of cut and tool nose radius on the cutting temperature and cutting force. Total 81 experiments have been carried out. 
The experimental results have been used to calculate Grey relational grade (GRG). Mathematical regression and ANN models are developed for the prediction of GRG and the predicted values are compared with calculated GRG. ANOVA tests have been carried out to evaluate contribution of parameters.

\section{Experimentation}

The following is the explanation of procedure adopted for the performance of experiments. Tool material, work piece material, instruments and tooling have been described here in this section.

\subsection{Workpiece and Tool}

The material used for experiment is Ti-6Al-4V ELI (round bar with 70mm diameter, 250mm length). The chemical composition of work material has been shown in Table 1.

\section{Table 1}

Chemical Composition of Ti-6Al-4V ELI

\begin{tabular}{ll}
\hline Element & Content (\%) \\
\hline Titanium, Ti & $88.09-91$ \\
Aluminum, Al & $5.5-6.5$ \\
Vanadium, V & $3.5-4.5$ \\
Iron, Fe & $\leq 0.25$ \\
Carbon, C & $\leq 0.080$ \\
Nitrogen, N & $\leq 0.030$ \\
Hydrogen, H & $\leq 0.0125$ \\
Other, each & $\leq 0.10$ \\
Other, total & $\leq 0.40$ \\
\hline
\end{tabular}

The cutting inserts which have been utilized are coated cemented carbide inserts with ISO designation as TNMG 160404, TNMG 160408 and TNMG 160412 with nose radius $0.4 \mathrm{~mm}, 0.8 \mathrm{~mm}$ and $1.2 \mathrm{~mm}$, respectively.

\subsection{Machining Tests}

All experiments were performed in dry environment using CNC turning center STC-200 with a maximum spindle speed of $3500 \mathrm{rpm}$ and a power rating of $9 \mathrm{KW}$. The maximum turning length of turning center was $400 \mathrm{~mm}$ and the maximum turning diameter was $200 \mathrm{~mm}$. The cutting forces have been measured using strain gauge type lathe tool dynamometer. The strain gauge type 3-channel lathe tool dynamometer was having resolution of $0.01 \mathrm{Kg}$ and accuracy of \pm 5 percent. The range of force was 0 to 200 in all three directions i.e. axial, radial and tangential. Cutting temperature was measured using MECO made infrared pyrometer (model IRT550P) for the range $-50^{\circ} \mathrm{C}$ to $500^{\circ} \mathrm{C}$. The surface roughness was measured by Mitutoyo SJ 410 having measuring range $800 \mu \mathrm{m} / 0.01 \mu \mathrm{m}$. Fig. 1 shows the machine tool, cutting tool and equipment used for the purpose of experimentation.

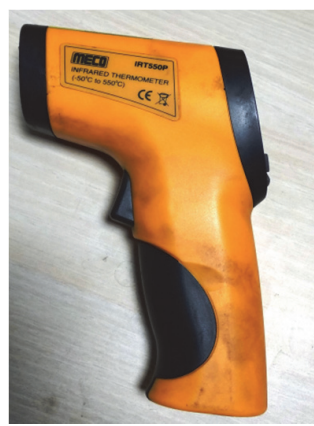

Fig. 1. (a) Infrared Pyrometer

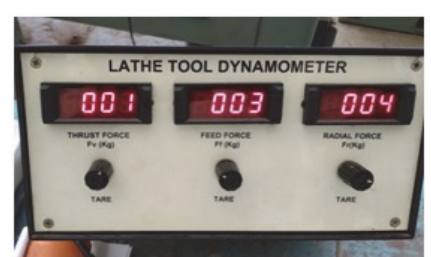

Fig. 1. (b) Lathe Tool Dynamometer

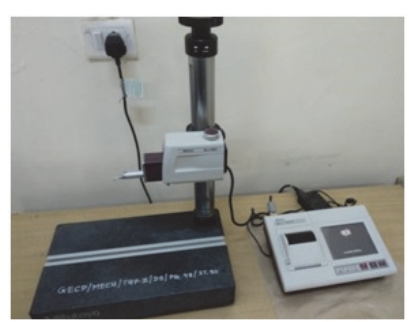

Fig. 1. (c) Surface Roughness Tester

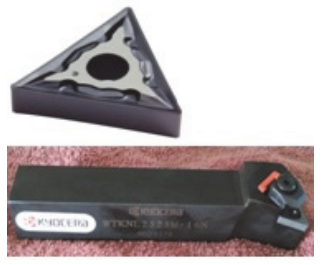

Fig. 1. (d) Cutting Tool and TNMG Insert 


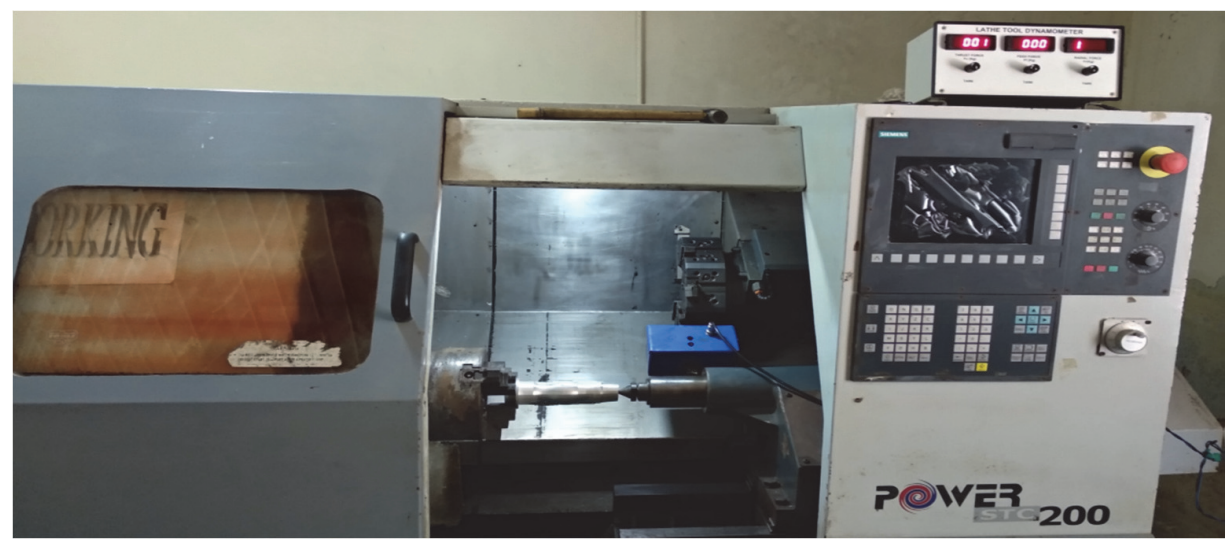

Fig. 1. (e) CNC lathe

Fig. 1. Machine and equipment used for experimentation

Four different cutting parameters have been chosen for experimentation. Cutting speed, feed and depth of cut are process parameters and tool nose radius is the parameter of cutting tool geometry. Table 2 indicates cutting parameters and their levels which have been set to carry out experiments. In this study, experiments have been planned for four different parameters with three levels. According to full factorial design for four parameters having 3 levels, total of $3^{4}=81$ experiments have been performed. The levels of parameters have been selected based on cutting tool supplier manual, trial runs of experiments and literature survey. The cutting parameters and measured responses have been presented in Table 3 . The effect of input parameters on responses like cutting force, cutting temperature and surface roughness were analyzed by main effect plots developed using Minitab-17. In order to investigate significance and contribution of individual parameters on multiple responses, Grey relational analysis is used; ANOVA test has been carried out on calculated Grey relational grade (GRG). ANOVA has also been utilized to model GRG. Regression equation is developed for the prediction of GRG. The effects of all input parameters on GRG are potted using 3D surface plots. The comparison of calculated and predicted values of GRG reveled the average error of $7.63 \%$. Using the measure responses values, ANN model was developed for the prediction of GRG. The ANN model predicted the values of GRG with average error of $3.75 \%$. The regression and ANN models are compared on a common graph.

\section{Table 2}

Level of input parameters

\begin{tabular}{lccc}
\hline Parameters & \multicolumn{3}{c}{ Levels } \\
\hline Feed $(\mathrm{mm})$ & 0.051 & 0.071 & 0.102 \\
Cutting Speed (rpm) & 140 & 224 & 315 \\
Depth of Cut (mm) & 0.5 & 0.75 & 1 \\
Tool Nose Radius $(\mathrm{mm})$ & 0.4 & 0.8 & 1.2 \\
\hline
\end{tabular}

Following steps are used for multi objective optimization using Grey Relational Analysis

1. The measured responses are normalized or preprocessed.

2. From normalized data, deviation sequence is determined.

3. Grey relational coefficient and Grey relational grade are obtained by calculations.

4. For statistical analysis of Grey relational grade, ANOVA tests are used.

5. Optimum parameters for turning are identified. 
Table 3

Full-Factorial Design of Experiments and Responses

\begin{tabular}{|c|c|c|c|c|c|c|c|}
\hline Exp No. & Nose Radius & Speed & Feed & Depth of Cut & Cutting Force & Temp. & Surface Roughness \\
\hline & $\begin{array}{c}\mathrm{Nr} \\
\mathrm{mm}\end{array}$ & $\begin{array}{c}\mathrm{Cs} \\
\mathrm{rpm}\end{array}$ & $\begin{array}{c}\mathrm{f} \\
\mathrm{mm} / \mathrm{rev}\end{array}$ & $\begin{array}{c}\mathrm{d} \\
\mathrm{mm}\end{array}$ & $\begin{array}{c}\mathrm{Fr} \\
\mathrm{Kg}\end{array}$ & $\begin{array}{c}\mathrm{T} \\
{ }^{0} \mathrm{C}\end{array}$ & $\begin{array}{l}\mathrm{Ra} \\
\mu \mathrm{m}\end{array}$ \\
\hline 1 & 0.4 & 140 & 0.051 & 0.5 & 16 & 53.7 & 1.271 \\
\hline 2 & 0.4 & 140 & 0.071 & 0.5 & 20 & 76.6 & 1.278 \\
\hline 3 & 0.4 & 140 & 0.102 & 0.5 & 22 & 81.2 & 1.282 \\
\hline 4 & 0.4 & 224 & 0.051 & 0.5 & 20 & 93.4 & 1.3 \\
\hline 5 & 0.4 & 224 & 0.071 & 0.5 & 21 & 106.8 & 1.495 \\
\hline 6 & 0.4 & 224 & 0.102 & 0.5 & 28 & 125.3 & 1.558 \\
\hline 7 & 0.4 & 315 & 0.051 & 0.5 & 27 & 84.1 & 1.601 \\
\hline 8 & 0.4 & 315 & 0.071 & 0.5 & 30 & 95.7 & 1.798 \\
\hline 9 & 0.4 & 315 & 0.102 & 0.5 & 31 & 102.5 & 1.832 \\
\hline 10 & 0.4 & 140 & 0.051 & 0.75 & 22 & 50.9 & 0.997 \\
\hline 11 & 0.4 & 140 & 0.071 & 0.75 & 26 & 78.2 & 1.001 \\
\hline 12 & 0.4 & 140 & 0.102 & 0.75 & 28 & 102.7 & 1.022 \\
\hline 13 & 0.4 & 224 & 0.051 & 0.75 & 23 & 65.2 & 1.041 \\
\hline 14 & 0.4 & 224 & 0.071 & 0.75 & 27 & 105.8 & 1.055 \\
\hline 15 & 0.4 & 224 & 0.102 & 0.75 & 28 & 121.1 & 1.081 \\
\hline 16 & 0.4 & 315 & 0.051 & 0.75 & 26 & 102.5 & 1.082 \\
\hline 17 & 0.4 & 315 & 0.071 & 0.75 & 27 & 116.2 & 1.115 \\
\hline 18 & 0.4 & 315 & 0.102 & 0.75 & 30 & 160.2 & 1.14 \\
\hline 19 & 0.4 & 140 & 0.051 & 1 & 32 & 46 & 1.141 \\
\hline 20 & 0.4 & 140 & 0.071 & 1 & 38 & 59.3 & 1.148 \\
\hline 21 & 0.4 & 140 & 0.102 & 1 & 39 & 99.6 & 1.155 \\
\hline 22 & 0.4 & 224 & 0.051 & 1 & 33 & 73.1 & 1.165 \\
\hline 23 & 0.4 & 224 & 0.071 & 1 & 38 & 85.8 & 1.169 \\
\hline 24 & 0.4 & 224 & 0.102 & 1 & 40 & 110.4 & 1.17 \\
\hline 25 & 0.4 & 315 & 0.051 & 1 & 41 & 90.8 & 1.172 \\
\hline 26 & 0.4 & 315 & 0.071 & 1 & 45 & 129 & 1.187 \\
\hline 27 & 0.4 & 315 & 0.102 & 1 & 71 & 156 & 1.255 \\
\hline 28 & 0.8 & 140 & 0.051 & 0.5 & 15 & 63.4 & 0.843 \\
\hline 29 & 0.8 & 140 & 0.071 & 0.5 & 18 & 105.9 & 0.853 \\
\hline 30 & 0.8 & 140 & 0.102 & 0.5 & 20 & 109.9 & 0.855 \\
\hline 31 & 0.8 & 224 & 0.051 & 0.5 & 19 & 52.5 & 0.858 \\
\hline 32 & 0.8 & 224 & 0.071 & 0.5 & 22 & 104.6 & 0.9 \\
\hline 33 & 0.8 & 224 & 0.102 & 0.5 & 23 & 132.5 & 0.904 \\
\hline 34 & 0.8 & 315 & 0.051 & 0.5 & 23 & 58 & 0.956 \\
\hline 35 & 0.8 & 315 & 0.071 & 0.5 & 24 & 74.5 & 0.991 \\
\hline 36 & 0.8 & 315 & 0.102 & 0.5 & 25 & 78.1 & 0.993 \\
\hline 37 & 0.8 & 140 & 0.051 & 0.75 & 23 & 57.6 & 0.618 \\
\hline 38 & 0.8 & 140 & 0.071 & 0.75 & 26 & 65.5 & 0.627 \\
\hline 39 & 0.8 & 140 & 0.102 & 0.75 & 30 & 71.4 & 0.631 \\
\hline 40 & 0.8 & 224 & 0.051 & 0.75 & 26 & 59.2 & 0.654 \\
\hline 41 & 0.8 & 224 & 0.071 & 0.75 & 29 & 83.4 & 0.674 \\
\hline 42 & 0.8 & 224 & 0.102 & 0.75 & 31 & 124 & 0.679 \\
\hline 43 & 0.8 & 315 & 0.051 & 0.75 & 33 & 95 & 0.681 \\
\hline 44 & 0.8 & 315 & 0.071 & 0.75 & 34 & 106.1 & 0.697 \\
\hline 45 & 0.8 & 315 & 0.102 & 0.75 & 38 & 139.9 & 0.711 \\
\hline 46 & 0.8 & 140 & 0.051 & 1 & 32 & 55 & 0.714 \\
\hline 47 & 0.8 & 140 & 0.071 & 1 & 42 & 58.7 & 0.729 \\
\hline 48 & 0.8 & 140 & 0.102 & 1 & 45 & 77 & 0.729 \\
\hline 49 & 0.8 & 224 & 0.051 & 1 & 42 & 62.9 & 0.733 \\
\hline 50 & 0.8 & 224 & 0.071 & 1 & 44 & 70.8 & 0.739 \\
\hline 51 & 0.8 & 224 & 0.102 & 1 & 45 & 78 & 0.752 \\
\hline 52 & 0.8 & 315 & 0.051 & 1 & 35 & 67.9 & 0.754 \\
\hline 53 & 0.8 & 315 & 0.071 & 1 & 37 & 114.7 & 0.786 \\
\hline 54 & 0.8 & 315 & 0.102 & 1 & 41 & 120 & 0.819 \\
\hline 55 & 1.2 & 140 & 0.051 & 0.5 & 12 & 79.9 & 0.533 \\
\hline 56 & 1.2 & 140 & 0.071 & 0.5 & 15 & 90.2 & 0.539 \\
\hline 57 & 1.2 & 140 & 0.102 & 0.5 & 17 & 109.9 & 0.55 \\
\hline 58 & 1.2 & 224 & 0.051 & 0.5 & 17 & 134.6 & 0.553 \\
\hline 59 & 1.2 & 224 & 0.071 & 0.5 & 18 & 138.2 & 0.578 \\
\hline 60 & 1.2 & 224 & 0.102 & 0.5 & 19 & 146.2 & 0.589 \\
\hline 61 & 1.2 & 315 & 0.051 & 0.5 & 18 & 129.1 & 0.594 \\
\hline 62 & 1.2 & 315 & 0.071 & 0.5 & 18 & 137.8 & 0.595 \\
\hline 63 & 1.2 & 315 & 0.102 & 0.5 & 18 & 138.8 & 0.596 \\
\hline 64 & 1.2 & 140 & 0.051 & 0.75 & 20 & 115.2 & 0.307 \\
\hline 65 & 1.2 & 140 & 0.071 & 0.75 & 22 & 121.6 & 0.329 \\
\hline 66 & 1.2 & 140 & 0.102 & 0.75 & 25 & 124.3 & 0.349 \\
\hline 67 & 1.2 & 224 & 0.051 & 0.75 & 25 & 68 & 0.364 \\
\hline 68 & 1.2 & 224 & 0.071 & 0.75 & 27 & 128.3 & 0.368 \\
\hline 69 & 1.2 & 224 & 0.102 & 0.75 & 30 & 166 & 0.406 \\
\hline 70 & 1.2 & 315 & 0.051 & 0.75 & 24 & 143 & 0.413 \\
\hline 71 & 1.2 & 315 & 0.071 & 0.75 & 28 & 156.7 & 0.415 \\
\hline 72 & 1.2 & 315 & 0.102 & 0.75 & 31 & 228.2 & 0.451 \\
\hline 73 & 1.2 & 140 & 0.051 & 1 & 35 & 95.7 & 0.47 \\
\hline 74 & 1.2 & 140 & 0.071 & 1 & 42 & 141.9 & 0.492 \\
\hline 75 & 1.2 & 140 & 0.102 & 1 & 49 & 181 & 0.503 \\
\hline 76 & 1.2 & 224 & 0.051 & 1 & 41 & 102.5 & 0.505 \\
\hline 77 & 1.2 & 224 & 0.071 & 1 & 44 & 110.9 & 0.508 \\
\hline 78 & 1.2 & 224 & 0.102 & 1 & 48 & 122.9 & 0.509 \\
\hline 79 & 1.2 & 315 & 0.051 & 1 & 47 & 162.6 & 0.509 \\
\hline 80 & 1.2 & 315 & 0.071 & 1 & 55 & 179 & 0.515 \\
\hline 81 & 1.2 & 315 & 0.102 & 1 & 57 & 219.5 & 0.529 \\
\hline
\end{tabular}

\subsection{Normalizing or Preprocessing of Data}

The measured responses were normalized by Grey relational method. The measured values of cutting force, cutting temperature and surface roughness were pre-processed to a sequence between zero and one. For normalizing in "higher-the-better" characteristic, the following equation is used. 


$$
x_{i}^{*}=\frac{x_{i}^{0}(k)-\min x_{i}^{0}(k)}{\max _{i}^{0}(k)-\min x_{i}^{0}(k)^{\prime}},
$$

and for "lower -the-better" characteristic, following equation is used.

$$
x_{i}^{*}=\frac{\operatorname{maxx}_{i}^{0}(k)-x_{i}^{0}(k)}{\max _{i}^{0}(k)-\min x_{i}^{0}(k)},
$$

where, $x_{i}^{0}=$ original value, $x_{i}^{*}=$ value after normalizing, $\max x_{i}^{0}=\operatorname{maximum}$ value of $x_{i}^{0}$ and $\min x_{i}^{*}=$ minimum value of $x_{i}^{0}$.

Here, in this study all the responses are required to be minimized; Eq. (2) is used for preprocessing/normalizing the data. The normalized data is shown in Table 4 .

\subsection{Grey Relational Coefficient and Grey Relational Grade}

After normalization the grey relational coefficient $\left(\mathbf{G R C} \xi_{\mathbf{i}}(\mathbf{k})\right)$ is calculated by Eq. (3) as follows,

$$
\xi_{i}(k)=\frac{\Delta_{\min }+\xi \Delta_{\max }}{\Delta_{0 i}(k)+\xi \Delta_{\max }},
$$

where, $\Delta_{\min }$ and $\Delta_{\max }$ are the maximum and minimum values in the normalized sequence, in this study they are 0 and 1 respectively. $\Delta_{0 i}(k)$ is the absolute difference between $x_{i}^{0}(k)$ and $x_{i}^{*}(\mathrm{k})$, for $\mathrm{i}=1$ to 81 and $\mathrm{k}=1$ to 3 , it is also named as deviation sequence. $\xi$ is coefficient of distinguishing, generally taken as 0.5. By averaging the values of GRC, Grey relational grade (GRG) $\gamma$ can be calculated by Eq. (4),

$$
\gamma=\frac{1}{n} \sum_{k=1}^{n} w_{k} \xi_{i}(k),
$$

where $w_{k}$ is normalized weight for response $\mathrm{k}$. Here in this study all responses are given equal weight, hence the Eq. (4) can be written as,

$$
\gamma=\frac{1}{n} \sum_{k=1}^{n} \xi_{i}(k) .
$$

The calculated values of GRC and GRG are tabulated in Table 4 
Table 4

Calculated Grey Relational Coefficient, Grey Relational Grade and Rank

\begin{tabular}{|c|c|c|c|c|c|c|c|c|}
\hline \multirow[t]{2}{*}{ Exp No. } & \multicolumn{3}{|c|}{ Normalized } & \multicolumn{3}{|c|}{$\mathrm{GRC}_{\mathrm{i}}(\mathbf{k})$} & $\operatorname{GRG}(\gamma)$ & Rank/ \\
\hline & Fr & $\mathbf{T}$ & $\mathbf{R a}$ & Fr & $\mathbf{T}$ & $\mathbf{R a}$ & & order \\
\hline 1 & 0.9322 & 0.9577 & 0.3679 & 0.8806 & 0.9221 & 0.4416 & 0.748 & 9 \\
\hline 2 & 0.8644 & 0.8321 & 0.3633 & 0.7867 & 0.7486 & 0.4399 & 0.658 & 36 \\
\hline 3 & 0.8305 & 0.8068 & 0.3607 & 0.7468 & 0.7213 & 0.4388 & 0.636 & 43 \\
\hline 4 & 0.8644 & 0.7398 & 0.3489 & 0.7867 & 0.6578 & 0.4343 & 0.626 & 47 \\
\hline 5 & 0.8475 & 0.6663 & 0.2210 & 0.7662 & 0.5997 & 0.3909 & 0.586 & 61 \\
\hline 6 & 0.7288 & 0.5648 & 0.1797 & 0.6484 & 0.5346 & 0.3787 & 0.521 & 76 \\
\hline 7 & 0.7458 & 0.7909 & 0.1515 & 0.6629 & 0.7051 & 0.3708 & 0.580 & 62 \\
\hline 8 & 0.6949 & 0.7272 & 0.0223 & 0.6211 & 0.6470 & 0.3384 & 0.535 & 73 \\
\hline 9 & 0.6780 & 0.6899 & 0.0000 & 0.6082 & 0.6172 & 0.3333 & 0.520 & 77 \\
\hline 10 & 0.8305 & 0.9731 & 0.5475 & 0.7468 & 0.9490 & 0.5250 & 0.740 & 11 \\
\hline 11 & 0.7627 & 0.8233 & 0.5449 & 0.6782 & 0.7388 & 0.5235 & 0.647 & 39 \\
\hline 12 & 0.7288 & 0.6888 & 0.5311 & 0.6484 & 0.6164 & 0.5161 & 0.594 & 59 \\
\hline 13 & 0.8136 & 0.8946 & 0.5187 & 0.7284 & 0.8259 & 0.5095 & 0.688 & 24 \\
\hline 14 & 0.7458 & 0.6718 & 0.5095 & 0.6629 & 0.6037 & 0.5048 & 0.590 & 60 \\
\hline 15 & 0.7288 & 0.5878 & 0.4925 & 0.6484 & 0.5481 & 0.4963 & 0.564 & 66 \\
\hline 16 & 0.7627 & 0.6899 & 0.4918 & 0.6782 & 0.6172 & 0.4959 & 0.597 & 55 \\
\hline 17 & 0.7458 & 0.6147 & 0.4702 & 0.6629 & 0.5648 & 0.4855 & 0.571 & 64 \\
\hline 18 & 0.6949 & 0.3732 & 0.4538 & 0.6211 & 0.4437 & 0.4779 & 0.514 & 78 \\
\hline 19 & 0.6610 & 1.0000 & 0.4531 & 0.5960 & 1.0000 & 0.4776 & 0.691 & 21 \\
\hline 20 & 0.5593 & 0.9270 & 0.4485 & 0.5315 & 0.8726 & 0.4755 & 0.627 & 46 \\
\hline 21 & 0.5424 & 0.7058 & 0.4439 & 0.5221 & 0.6296 & 0.4735 & 0.542 & 72 \\
\hline 22 & 0.6441 & 0.8513 & 0.4374 & 0.5842 & 0.7707 & 0.4705 & 0.608 & 52 \\
\hline 23 & 0.5593 & 0.7816 & 0.4348 & 0.5315 & 0.6960 & 0.4694 & 0.566 & 65 \\
\hline 24 & 0.5254 & 0.6465 & 0.4341 & 0.5130 & 0.5859 & 0.4691 & 0.523 & 75 \\
\hline 25 & 0.5085 & 0.7541 & 0.4328 & 0.5043 & 0.6703 & 0.4685 & 0.548 & 70 \\
\hline 26 & 0.4407 & 0.5445 & 0.4230 & 0.4720 & 0.5233 & 0.4642 & 0.486 & 80 \\
\hline 27 & 0.0000 & 0.3963 & 0.3784 & 0.3333 & 0.4530 & 0.4458 & 0.411 & 81 \\
\hline 28 & 0.9492 & 0.9045 & 0.6485 & 0.9077 & 0.8396 & 0.5872 & 0.778 & 5 \\
\hline 29 & 0.8983 & 0.6712 & 0.6420 & 0.8310 & 0.6033 & 0.5827 & 0.672 & 30 \\
\hline 30 & 0.8644 & 0.6493 & 0.6407 & 0.7867 & 0.5877 & 0.5818 & 0.652 & 38 \\
\hline 31 & 0.8814 & 0.9643 & 0.6387 & 0.8082 & 0.9334 & 0.5805 & 0.774 & 7 \\
\hline 32 & 0.8305 & 0.6784 & 0.6111 & 0.7468 & 0.6086 & 0.5625 & 0.639 & 41 \\
\hline 33 & 0.8136 & 0.5252 & 0.6085 & 0.7284 & 0.5130 & 0.5609 & 0.601 & 54 \\
\hline 34 & 0.8136 & 0.9341 & 0.5744 & 0.7284 & 0.8836 & 0.5402 & 0.717 & 16 \\
\hline 35 & 0.7966 & 0.8436 & 0.5515 & 0.7108 & 0.7617 & 0.5271 & 0.667 & 32 \\
\hline 36 & 0.7797 & 0.8238 & 0.5502 & 0.6941 & 0.7394 & 0.5264 & 0.653 & 37 \\
\hline 37 & 0.8136 & 0.9363 & 0.7961 & 0.7284 & 0.8870 & 0.7103 & 0.775 & 6 \\
\hline 38 & 0.7627 & 0.8930 & 0.7902 & 0.6782 & 0.8237 & 0.7044 & 0.735 & 12 \\
\hline 39 & 0.6949 & 0.8606 & 0.7875 & 0.6211 & 0.7820 & 0.7018 & 0.702 & 19 \\
\hline 40 & 0.7627 & 0.9276 & 0.7725 & 0.6782 & 0.8734 & 0.6872 & 0.746 & 10 \\
\hline 41 & 0.7119 & 0.7947 & 0.7593 & 0.6344 & 0.7089 & 0.6751 & 0.673 & 28 \\
\hline 42 & 0.6780 & 0.5719 & 0.7561 & 0.6082 & 0.5387 & 0.6721 & 0.606 & 53 \\
\hline 43 & 0.6441 & 0.7311 & 0.7548 & 0.5842 & 0.6502 & 0.6709 & 0.635 & 44 \\
\hline 44 & 0.6271 & 0.6701 & 0.7443 & 0.5728 & 0.6025 & 0.6616 & 0.612 & 51 \\
\hline 45 & 0.5593 & 0.4846 & 0.7351 & 0.5315 & 0.4924 & 0.6537 & 0.559 & 68 \\
\hline 46 & 0.6610 & 0.9506 & 0.7331 & 0.5960 & 0.9101 & 0.6520 & 0.719 & 15 \\
\hline 47 & 0.4915 & 0.9303 & 0.7233 & 0.4958 & 0.8776 & 0.6437 & 0.672 & 29 \\
\hline 48 & 0.4407 & 0.8299 & 0.7233 & 0.4720 & 0.7461 & 0.6437 & 0.621 & 48 \\
\hline 49 & 0.4915 & 0.9072 & 0.7207 & 0.4958 & 0.8435 & 0.6416 & 0.660 & 34 \\
\hline 50 & 0.4576 & 0.8639 & 0.7167 & 0.4797 & 0.7860 & 0.6383 & 0.635 & 45 \\
\hline 51 & 0.4407 & 0.8244 & 0.7082 & 0.4720 & 0.7400 & 0.6315 & 0.615 & 50 \\
\hline 52 & 0.6102 & 0.8798 & 0.7069 & 0.5619 & 0.8062 & 0.6304 & 0.666 & 33 \\
\hline 53 & 0.5763 & 0.6229 & 0.6859 & 0.5413 & 0.5701 & 0.6142 & 0.575 & 63 \\
\hline 54 & 0.5085 & 0.5939 & 0.6643 & 0.5043 & 0.5518 & 0.5983 & 0.551 & 69 \\
\hline 55 & 1.0000 & 0.8139 & 0.8518 & 1.0000 & 0.7288 & 0.7714 & 0.833 & 1 \\
\hline 56 & 0.9492 & 0.7574 & 0.8479 & 0.9077 & 0.6733 & 0.7667 & 0.783 & 4 \\
\hline 57 & 0.9153 & 0.6493 & 0.8407 & 0.8551 & 0.5877 & 0.7583 & 0.734 & 13 \\
\hline 58 & 0.9153 & 0.5137 & 0.8387 & 0.8551 & 0.5070 & 0.7561 & 0.706 & 17 \\
\hline 59 & 0.8983 & 0.4940 & 0.8223 & 0.8310 & 0.4970 & 0.7378 & 0.689 & 23 \\
\hline 60 & 0.8814 & 0.4501 & 0.8151 & 0.8082 & 0.4762 & 0.7300 & 0.671 & 31 \\
\hline 61 & 0.8983 & 0.5439 & 0.8118 & 0.8310 & 0.5230 & 0.7265 & 0.693 & 20 \\
\hline 62 & 0.8983 & 0.4962 & 0.8111 & 0.8310 & 0.4981 & 0.7258 & 0.685 & 25 \\
\hline 63 & 0.8983 & 0.4907 & 0.8105 & 0.8310 & 0.4954 & 0.7252 & 0.684 & 26 \\
\hline 64 & 0.8644 & 0.6202 & 1.0000 & 0.7867 & 0.5683 & 1.0000 & 0.785 & 3 \\
\hline 65 & 0.8305 & 0.5851 & 0.9856 & 0.7468 & 0.5465 & 0.9720 & 0.755 & 8 \\
\hline 66 & 0.7797 & 0.5703 & 0.9725 & 0.6941 & 0.5378 & 0.9478 & 0.727 & 14 \\
\hline 67 & 0.7797 & 0.8793 & 0.9626 & 0.6941 & 0.8055 & 0.9304 & 0.810 & 2 \\
\hline 68 & 0.7458 & 0.5483 & 0.9600 & 0.6629 & 0.5254 & 0.9259 & 0.705 & 18 \\
\hline 69 & 0.6949 & 0.3414 & 0.9351 & 0.6211 & 0.4315 & 0.8851 & 0.646 & 40 \\
\hline 70 & 0.7966 & 0.4676 & 0.9305 & 0.7108 & 0.4843 & 0.8780 & 0.691 & 22 \\
\hline 71 & 0.7288 & 0.3924 & 0.9292 & 0.6484 & 0.4514 & 0.8759 & 0.659 & 35 \\
\hline 72 & 0.6780 & 0.0000 & 0.9056 & 0.6082 & 0.3333 & 0.8411 & 0.594 & 58 \\
\hline 73 & 0.6102 & 0.7272 & 0.8931 & 0.5619 & 0.6470 & 0.8239 & 0.678 & 27 \\
\hline 74 & 0.4915 & 0.4737 & 0.8787 & 0.4958 & 0.4872 & 0.8047 & 0.596 & 56 \\
\hline 75 & 0.3729 & 0.2591 & 0.8715 & 0.4436 & 0.4029 & 0.7955 & 0.547 & 71 \\
\hline 76 & 0.5085 & 0.6899 & 0.8702 & 0.5043 & 0.6172 & 0.7939 & 0.638 & 42 \\
\hline 77 & 0.4576 & 0.6438 & 0.8682 & 0.4797 & 0.5840 & 0.7914 & 0.618 & 49 \\
\hline 78 & 0.3898 & 0.5779 & 0.8675 & 0.4504 & 0.5423 & 0.7906 & 0.594 & 57 \\
\hline 79 & 0.4068 & 0.3600 & 0.8675 & 0.4574 & 0.4386 & 0.7906 & 0.562 & 67 \\
\hline 80 & 0.2712 & 0.2700 & 0.8636 & 0.4069 & 0.4065 & 0.7857 & 0.533 & 74 \\
\hline 81 & 0.2373 & 0.0477 & 0.8544 & 0.3960 & 0.3443 & 0.7745 & 0.505 & 79 \\
\hline
\end{tabular}

\section{Analysis and discussion}

\subsection{Effect of Parameters on Responses}

In present study multiple response like cutting force, average cutting temperature and surface roughness were optimized for turning of Ti-6Al-4V (ELI). The influence of parameters cutting speed, feed, depth of cut and tool nose radius is analyzed. 


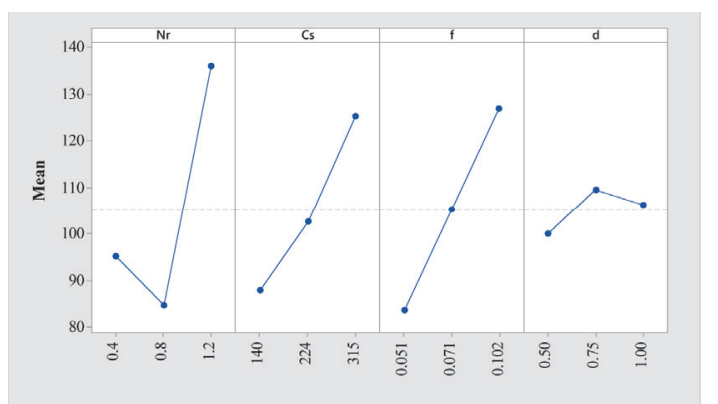

Fig. 2(a). Mean effect plots for Temperature

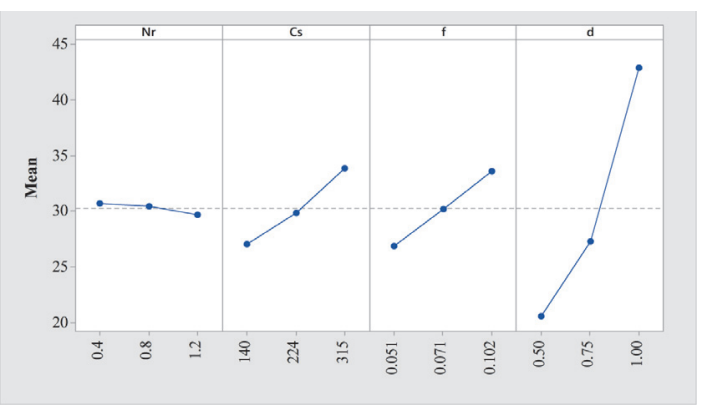

Fig. 2(b). Mean effect plots for Cutting Force

The mean effect plots for cutting parameters on temperature, cutting force and surface roughness are shown in Fig. 2(a), Fig. 2(b) and Fig. 2(c) respectively. From Fig. 2(a) it can be observed that increase in tool nose radius initially decreases the temperature and then the temperature increases with increase in nose radius. With the increase in cutting speed and feed the average cutting temperature rises. The depth of cut has lesser influence of cutting temperature. From Fig. 2(b), it can be interpreted that nose radius is having least influence on cutting force. The cutting force increases with increase of cutting speed and feed rate. The depth of cut is the maximum influence on cutting force while turning.

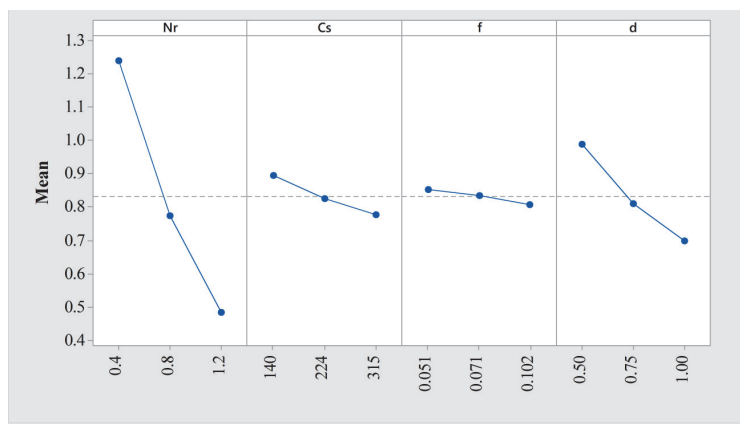

Fig. 2(c). Mean effect plots for Surface Roughness

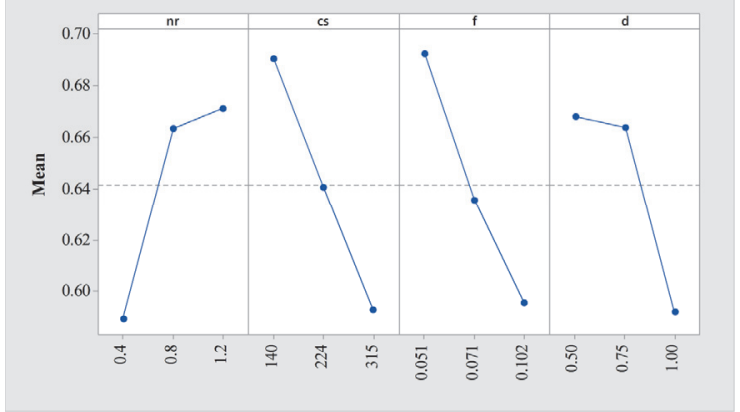

Fig. 3(a). Mean effect plots for Grey relational grade

Fig. 2(c) shows that surface roughness is highly influenced by tool nose radius. The increase in nose radius highly decreases the surface roughness. The effect on cutting force with change in depth of cut is high as compared to change in cutting speed and feed.

\subsection{ANOVA and Grey Relational Analysis}

Many researchers have worked on single objective optimization. In this study multi objective optimization is done using Grey relational Analysis. From Table 5, it is clear that the experiment number 55 is having maximum GRG value of 0.833 . The process parameters for experiment number 55 are cutting speed as $140 \mathrm{rpm}$, Nose radius $1.2 \mathrm{~mm}$, Feed $0.051 \mathrm{~mm} / \mathrm{rev}$ and depth of cut is $0.5 \mathrm{~mm}$. These values of parameters are considered as optimum process parameters for turning Ti-6Al-4V (ELI) among the 81 experiments. The influence of cutting parameters on Grey Relational Grade are analyzed (See Fig. 3). In Fig. 3(a), effect of parameters on Grey relational grade is shown. It can be said from Fig. 3(a) that feed and cutting speed are significant factors for multiple responses when studied simultaneously. Increase in nose radius increases grey relational grade whereas it is decreased with increase in depth of cut. ANOVA tests were performed for statistical analysis of effect of turning parameters on Grey relational Grade. Table 7 shows the results of ANOVA tests. It can be interpreted from Table 7 that Feed rate is having maximum influence followed by cutting speed, nose radius and depth of cut. The R-Square value for the model developed for GRG is $88.74 \%$ 
Table 7

ANOVA test for GRG

\begin{tabular}{ccccccc}
\hline Source & DF & Adi SS & Adi MS & F-Value & P-Value & \% contribution \\
\hline $\mathrm{nr}$ & 1 & 0.092466 & 0.092466 & 98.1 & 0.000 & 18.74 \\
$\mathrm{cs}$ & 1 & 0.125319 & 0.125319 & 132.96 & 0.000 & 25.40 \\
$\mathrm{f}$ & 1 & 0.125633 & 0.125633 & 133.29 & 0.000 & 25.46 \\
$\mathrm{~d}$ & 1 & 0.078392 & 0.078392 & 83.17 & 0.000 & 15.89 \\
$\mathrm{nr} \times \mathrm{nr}$ & 1 & 0.019742 & 0.019742 & 20.95 & 0.000 & 4.00 \\
$\mathrm{cs} \times \mathrm{cs}$ & 1 & 0.000157 & 0.000157 & 0.17 & 0.684 & 0.03 \\
$\mathrm{f} \times \mathrm{f}$ & 1 & 0.006146 & 0.006146 & 6.52 & 0.013 & 1.25 \\
$\mathrm{~d} \times \mathrm{d}$ & 1 & 0.020297 & 0.020297 & 21.53 & 0.000 & 4.11 \\
$\mathrm{nr} \times \mathrm{cs}$ & 1 & 0.00228 & 0.00228 & 2.42 & 0.125 & 0.46 \\
$\mathrm{n} \times \mathrm{f}$ & 1 & 0.002537 & 0.002537 & 2.69 & 0.106 & 0.51 \\
$\mathrm{nr} \times \mathrm{d}$ & 1 & 0.01768 & 0.01768 & 18.76 & 0.000 & 3.58 \\
$\mathrm{cs} \times \mathrm{f}$ & 1 & 0.002238 & 0.002238 & 2.37 & 0.128 & 0.45 \\
$\mathrm{cs} \times \mathrm{d}$ & 1 & 0.000323 & 0.000323 & 0.34 & 0.560 & 0.06 \\
$\mathrm{f} \times \mathrm{d}$ & 1 & 0.000245 & 0.000245 & 0.26 & 0.612 & 0.05 \\
Error & 66 & 0.062209 & 0.000943 & & & \\
\hline $\mathrm{T}$ tal & 80 & 0.552357 & & 523.53 & & 100 \\
\hline
\end{tabular}

For better understating the effect of input parameters on Grey relational Grade, 3D surface plots are obtained. The effect of feed and depth of cut on GRG is shown in figure 3(b). Increase in feed rated reduces the GRG value. The GRG value is initially increases and highly decreased with increase in depth of cut. Fig. 3(c) shows the effect of nose radius and cutting speed on GRG. The increase in cutting speed makes the GRG to be decrease. The GRG value highly increases by increase in nose radius.

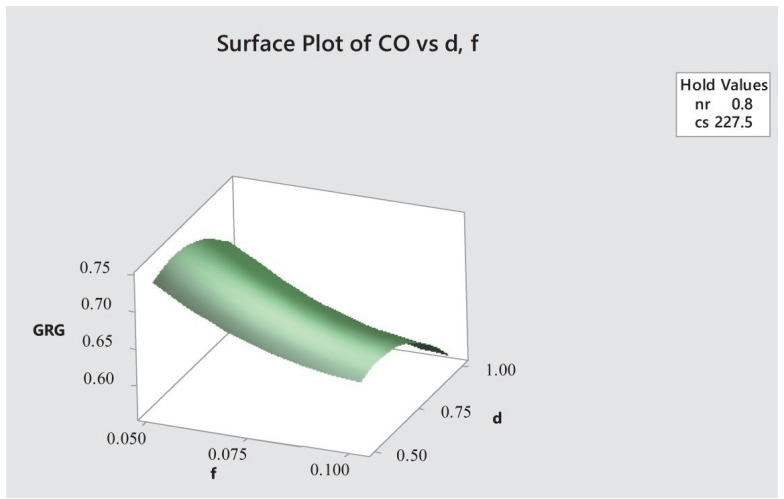

Fig. 3(b). Surface plot of Grey relational grade v/s feed and depth of cut

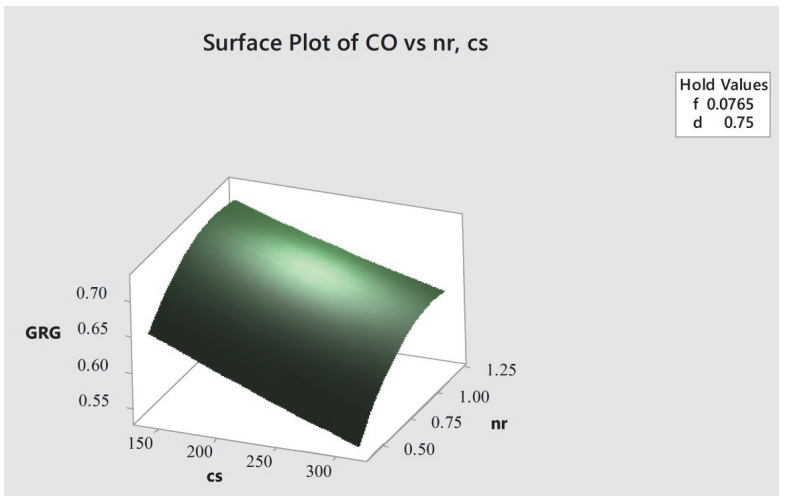

Fig. 3(c). Surface plot of Grey relational grade $\mathrm{v} / \mathrm{s}$ cutting speed and nose radius

For analysis of effect of influence of parameters on GRG, the average grey relational grades are obtained. The mean response values for GRG are tabulated in Table 6

Table 6

Mean response table for Grey relational grade

\begin{tabular}{ccccc}
\hline Level & Nose Radius & Speed & Feed & Depth of Cut \\
\hline 1 & 0.589487 & 0.690618 & 0.692441 & 0.668197 \\
2 & 0.663443 & 0.640698 & 0.635902 & 0.663751 \\
3 & 0.671164 & 0.592778 & 0.595751 & 0.592146 \\
\hline Delta & 0.007721 & 0.09784 & 0.09669 & 0.076051 \\
Rank & 4 & 1 & 2 & 3 \\
\hline
\end{tabular}

From Table 6, it is clear that the cutting speed is significant factor while considering multiple responses simultaneously. The feed, depth of cut and nose radius are having influence in decreasing order. Pareto 
chart is prepared to analyze the contribution of cutting parameters on multiple responses. Figure 4 shows the Pareto chart for machining parameters on GRG value. It can be interpreted that feed and cutting speed pay significant contribution on measured responses like cutting temperature, cutting force and surface roughness while optimizing simultaneously.

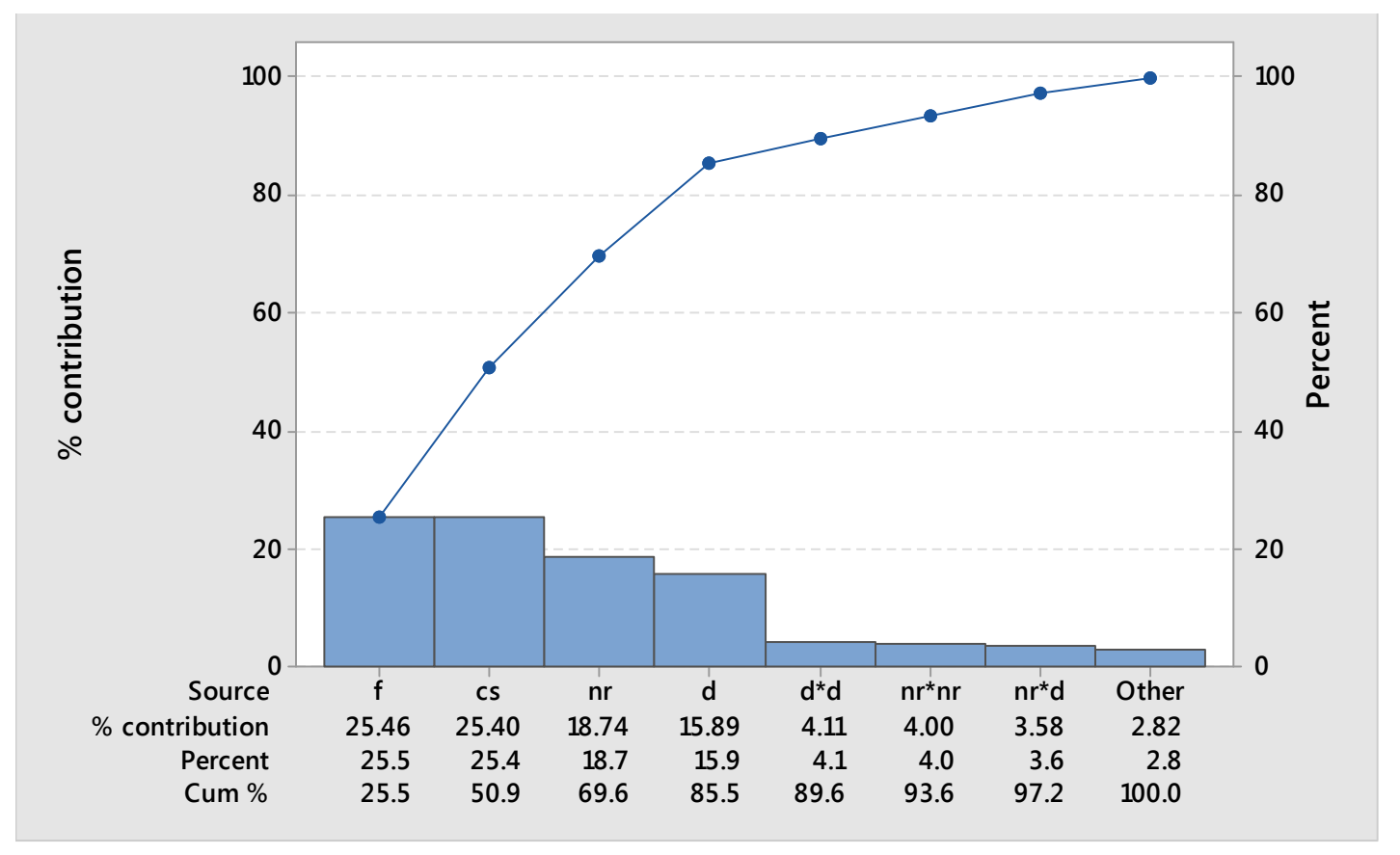

Fig. 4. Pareto chart for machining parameters

The regression equation for Grey relational grade is obtained using Minitab software. The equation is as follows:

$$
\begin{aligned}
& \mathrm{GRG}=0.702+0.4871 \mathrm{Nr}-0.001076 \mathrm{Cs}-7.63 \mathrm{f}+0.892 \mathrm{~d}-0.2070 \mathrm{Nr} \times \mathrm{Nr}+30.0 \mathrm{f} \times \mathrm{f} \\
& -0.537 \mathrm{~d} \times \mathrm{d}+0.000227 \mathrm{Nr} \times \mathrm{Cs}+0.817 \mathrm{Nr} \times \mathrm{f}-0.2216 \mathrm{Nr} \times \mathrm{d}+0.00351 \mathrm{Cs} \times \mathrm{f} \\
& -0.000137 \mathrm{Cs} \times \mathrm{d}-0.406 \mathrm{f} \times \mathrm{d}
\end{aligned}
$$

Using Eq. (6), the GRG values are predicted and compared with calculated values of GRG. The comparison is shown in Table 5. The average error is $7.63 \%$.

\subsection{Artificial Neural Network}

In order to develop more precise model, the artificial neural network is used. Initially input data, sample data and corresponding output data are created in Matlab workspace. Using these data a network is created in workspace. In present study, the Feed forward back propagation model has been used. From the data fed in the workspace, $75 \%$ are used for training, $12 \%$ for testing and $12 \%$ for validation purpose. 'TRAINLM' function was used for training and 'PURELIN' function was used as transfer function. Then the developed network was ready for training. The training was done until the predicted value matches nearer to the actual experimental values. The graphs for mean square error values for training, testing, validation and overall target data are shown in Figure 5. The predicted values of GRG by ANN are compared with calculated GRG values and are tabulated in Table 6 . The average error is $3.75 \%$. The fitness for ANN model is $95.54 \%$. 
Table 5

Comparison of calculated GRG and predicted GRG

\begin{tabular}{|c|c|c|c|c|c|c|c|}
\hline Exp No. & GRG & Re-GRG & Error (\%) & Exp No. & GRG & Re-GRG & Error (\%) \\
\hline 1 & 0.748 & 0.710 & 5.39 & 42 & 0.705 & 0.756 & 6.77 \\
\hline 2 & 0.778 & 0.857 & 9.23 & 43 & 0.571 & 0.587 & 2.66 \\
\hline 3 & 0.833 & 0.855 & 2.54 & 44 & 0.612 & 0.719 & 14.84 \\
\hline 4 & 0.626 & 0.669 & 6.45 & 45 & 0.659 & 0.702 & 6.16 \\
\hline 5 & 0.774 & 0.826 & 6.31 & 46 & 0.594 & 0.653 & 9.14 \\
\hline 6 & 0.706 & 0.833 & 15.29 & 47 & 0.702 & 0.773 & 9.27 \\
\hline 7 & 0.580 & 0.610 & 4.94 & 48 & 0.727 & 0.744 & 2.31 \\
\hline 8 & 0.717 & 0.777 & 7.63 & 49 & 0.564 & 0.592 & 4.64 \\
\hline 9 & 0.693 & 0.794 & 12.66 & 50 & 0.606 & 0.721 & 15.91 \\
\hline 10 & 0.658 & 0.657 & 0.16 & 51 & 0.646 & 0.701 & 7.84 \\
\hline 11 & 0.672 & 0.809 & 16.93 & 52 & 0.514 & 0.509 & 1.02 \\
\hline 12 & 0.783 & 0.812 & 3.61 & 53 & 0.559 & 0.649 & 13.77 \\
\hline 13 & 0.586 & 0.612 & 4.31 & 54 & 0.594 & 0.638 & 6.93 \\
\hline 14 & 0.639 & 0.773 & 17.34 & 55 & 0.691 & 0.735 & 5.92 \\
\hline 15 & 0.689 & 0.785 & 12.32 & 56 & 0.719 & 0.804 & 10.48 \\
\hline 16 & 0.535 & 0.547 & 2.11 & 57 & 0.678 & 0.723 & 6.27 \\
\hline 17 & 0.667 & 0.719 & 7.24 & 58 & 0.608 & 0.677 & 10.10 \\
\hline 18 & 0.685 & 0.741 & 7.51 & 59 & 0.660 & 0.755 & 12.55 \\
\hline 19 & 0.636 & 0.613 & 3.71 & 60 & 0.638 & 0.684 & 6.63 \\
\hline 20 & 0.652 & 0.772 & 15.55 & 61 & 0.548 & 0.598 & 8.44 \\
\hline 21 & 0.734 & 0.782 & 6.16 & 62 & 0.666 & 0.687 & 2.98 \\
\hline 22 & 0.521 & 0.560 & 7.05 & 63 & 0.562 & 0.626 & 10.12 \\
\hline 23 & 0.601 & 0.729 & 17.55 & 64 & 0.627 & 0.660 & 5.02 \\
\hline 24 & 0.671 & 0.748 & 10.19 & 65 & 0.672 & 0.733 & 8.29 \\
\hline 25 & 0.520 & 0.487 & 6.74 & 66 & 0.596 & 0.657 & 9.32 \\
\hline 26 & 0.653 & 0.666 & 1.84 & 67 & 0.566 & 0.597 & 5.24 \\
\hline 27 & 0.684 & 0.695 & 1.57 & 68 & 0.635 & 0.680 & 6.64 \\
\hline 28 & 0.740 & 0.779 & 4.97 & 69 & 0.618 & 0.613 & 0.85 \\
\hline 29 & 0.775 & 0.887 & 12.61 & 70 & 0.486 & 0.513 & 5.16 \\
\hline 30 & 0.785 & 0.846 & 7.18 & 71 & 0.575 & 0.606 & 5.09 \\
\hline 31 & 0.688 & 0.730 & 5.74 & 72 & 0.533 & 0.550 & 3.01 \\
\hline 32 & 0.746 & 0.847 & 11.93 & 73 & 0.542 & 0.580 & 6.66 \\
\hline 33 & 0.810 & 0.815 & 0.65 & 74 & 0.621 & 0.661 & 6.12 \\
\hline 34 & 0.597 & 0.661 & 9.62 & 75 & 0.547 & 0.592 & 7.58 \\
\hline 35 & 0.635 & 0.788 & 19.44 & 76 & 0.523 & 0.510 & 2.48 \\
\hline 36 & 0.691 & 0.766 & 9.84 & 77 & 0.615 & 0.600 & 2.40 \\
\hline 37 & 0.647 & 0.715 & 9.56 & 78 & 0.594 & 0.541 & 9.94 \\
\hline 38 & 0.735 & 0.828 & 11.18 & 79 & 0.411 & 0.418 & 1.73 \\
\hline 39 & 0.755 & 0.791 & 4.56 & 80 & 0.551 & 0.518 & 6.43 \\
\hline 40 & 0.590 & 0.661 & 10.69 & 81 & 0.505 & 0.469 & 7.69 \\
\hline 41 & 0.673 & 0.783 & 14.11 & & & & \\
\hline
\end{tabular}

Table 6

Comparison of calculated GRG and ANN predicted GRG

\begin{tabular}{|c|c|c|c|c|c|c|c|}
\hline Exp No. & GRG & ANN GRG & Error (\%) & Exp No. & GRG & ANN GRG & Error (\%) \\
\hline 1 & 0.748 & 0.729 & 2.67 & 42 & 0.705 & 2.74 & 2.74 \\
\hline 2 & 0.778 & 0.665 & 0.99 & 43 & 0.571 & 4.07 & 4.07 \\
\hline 3 & 0.833 & 0.634 & 0.21 & 44 & 0.612 & 4.81 & 4.81 \\
\hline 4 & 0.626 & 0.711 & 11.97 & 45 & 0.659 & 0.70 & 0.70 \\
\hline 5 & 0.774 & 0.585 & 0.14 & 46 & 0.594 & 0.41 & 0.41 \\
\hline 6 & 0.706 & 0.514 & 1.34 & 47 & 0.702 & 2.10 & 2.10 \\
\hline 7 & 0.580 & 0.608 & 4.70 & 48 & 0.727 & 2.21 & 2.21 \\
\hline 8 & 0.717 & 0.535 & 0.01 & 49 & 0.564 & 2.92 & 2.92 \\
\hline 9 & 0.693 & 0.543 & 4.37 & 50 & 0.606 & 12.87 & 12.87 \\
\hline 10 & 0.658 & 0.725 & 2.15 & 51 & 0.646 & 1.01 & 1.01 \\
\hline 11 & 0.672 & 0.650 & 0.51 & 52 & 0.514 & 5.50 & 5.50 \\
\hline 12 & 0.783 & 0.605 & 1.96 & 53 & 0.559 & 7.29 & 7.29 \\
\hline 13 & 0.586 & 0.675 & 1.93 & 54 & 0.594 & 1.19 & 1.19 \\
\hline 14 & 0.639 & 0.537 & 9.92 & 55 & 0.691 & 0.94 & 0.94 \\
\hline 15 & 0.689 & 0.543 & 3.97 & 56 & 0.719 & 0.17 & 0.17 \\
\hline 16 & 0.535 & 0.604 & 1.21 & 57 & 0.678 & 9.20 & 9.20 \\
\hline 17 & 0.667 & 0.567 & 0.68 & 58 & 0.608 & 13.97 & 13.97 \\
\hline 18 & 0.685 & 0.522 & 1.56 & 59 & 0.660 & 6.88 & 6.88 \\
\hline 19 & 0.636 & 0.687 & 0.55 & 60 & 0.638 & 8.74 & 8.74 \\
\hline 20 & 0.652 & 0.668 & 6.23 & 61 & 0.548 & 3.84 & 3.84 \\
\hline 21 & 0.734 & 0.542 & 0.11 & 62 & 0.666 & 1.44 & 1.44 \\
\hline 22 & 0.521 & 0.606 & 0.48 & 63 & 0.562 & 4.37 & 4.37 \\
\hline 23 & 0.601 & 0.549 & 3.05 & 64 & 0.627 & 0.58 & 0.58 \\
\hline 24 & 0.671 & 0.527 & 0.76 & 65 & 0.672 & 1.17 & 1.17 \\
\hline 25 & 0.520 & 0.579 & 5.37 & 66 & 0.596 & 5.81 & 5.81 \\
\hline 26 & 0.653 & 0.517 & 5.97 & 67 & 0.566 & 2.55 & 2.55 \\
\hline 27 & 0.684 & 0.483 & 15.01 & 68 & 0.635 & 3.06 & 3.06 \\
\hline 28 & 0.740 & 0.801 & 2.86 & 69 & 0.618 & 1.81 & 1.81 \\
\hline 29 & 0.775 & 0.743 & 9.56 & 70 & 0.486 & 0.41 & 0.41 \\
\hline 30 & 0.785 & 0.703 & 7.19 & 71 & 0.575 & 2.28 & 2.28 \\
\hline 31 & 0.688 & 0.771 & 0.38 & 72 & 0.533 & 2.08 & 2.08 \\
\hline 32 & 0.746 & 0.667 & 4.11 & 73 & 0.542 & 8.66 & 8.66 \\
\hline 33 & 0.810 & 0.607 & 1.06 & 74 & 0.621 & 17.88 & 17.88 \\
\hline 34 & 0.597 & 0.679 & 5.69 & 75 & 0.547 & 6.72 & 6.72 \\
\hline 35 & 0.635 & 0.642 & 3.91 & 76 & 0.523 & 2.70 & 2.70 \\
\hline 36 & 0.691 & 0.644 & 1.40 & 77 & 0.615 & 8.05 & 8.05 \\
\hline 37 & 0.647 & 0.779 & 0.51 & 78 & 0.594 & 4.13 & 4.13 \\
\hline 38 & 0.735 & 0.723 & 1.68 & 79 & 0.411 & 5.13 & 5.13 \\
\hline 39 & 0.755 & 0.693 & 1.25 & 80 & 0.551 & 0.59 & 0.59 \\
\hline 40 & 0.590 & 0.751 & 0.63 & 81 & 0.505 & 0.78 & 0.78 \\
\hline 41 & 0.673 & 0.647 & 2.67 & & & & \\
\hline
\end{tabular}



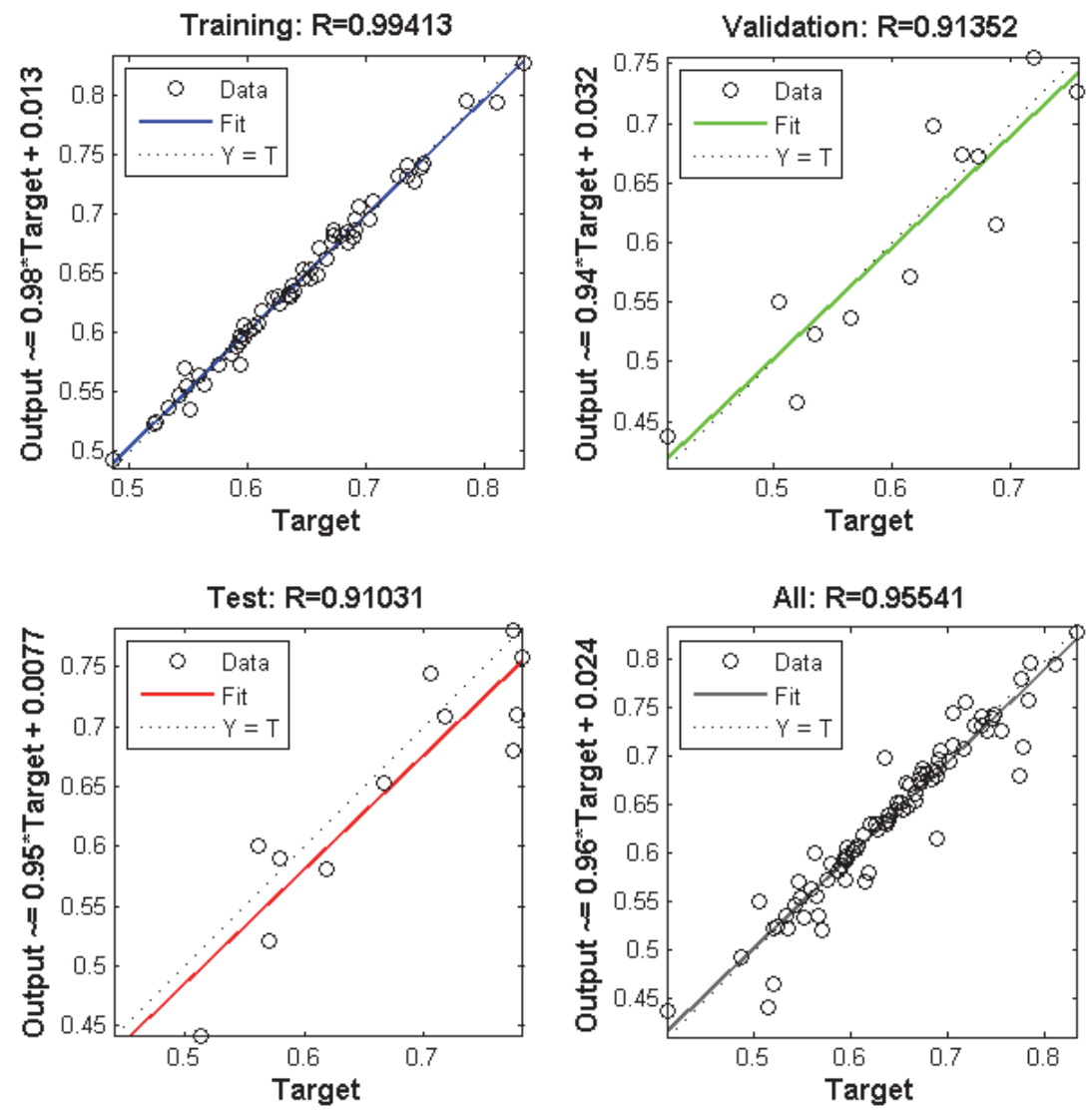

Fig. 5. Training, Validation, Test and overall target Graph

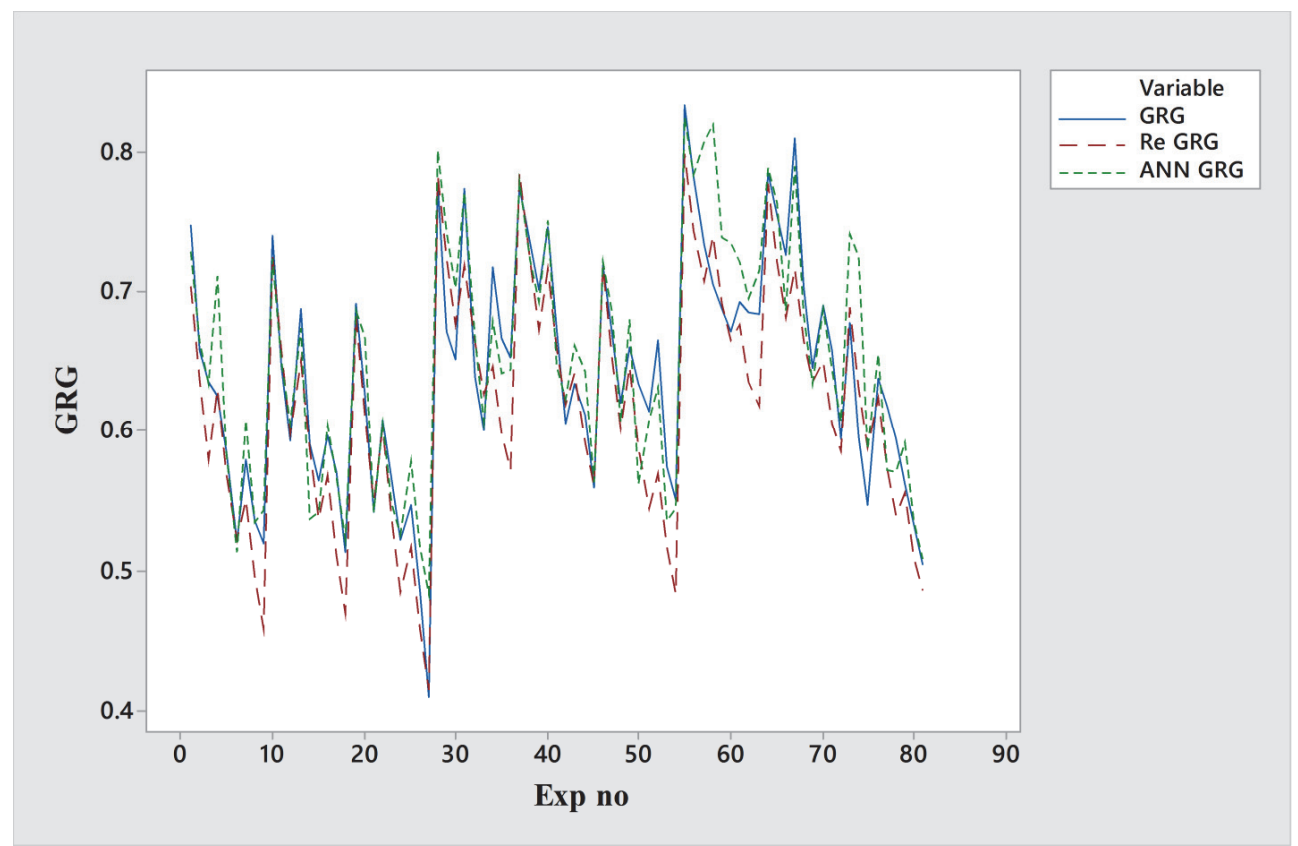

Fig. 6. Comparative graph for calculated GRG v/s predicted values of GRG

The models developed for GRG using Regression and ANN are compared and shown in Fig. 6. The calculated GRG, Re-GRG and ANN GRG are shown in comparative graph. It can be easily interpreted 
that ANN model predict the response more precisely as compared to Regression model. The Graph line of ANN GRG values are almost merged with calculated GRG values. Hence it can be said that ANN model can be used when the precision of model is utmost requirement.

\section{Conclusion}

The effects of cutting parameters like cutting speed, feed, depth of cut and tool nose radius on responses like cutting temperature, cutting force and surface roughness have been investigated for Ti-6Al-4V ELI in present study. Following points have been concluded from the experimental study which has been carried out.

- For multi objective optimization, Grey Relational Analysis was used.

- From experimental data, mathematical models are developed for Grey Relational Grade using Regression method and Artificial Neural Network method.

- The optimum parameters to minimize cutting force, cutting temperature and surface roughness while turning Ti-6Al-4V (ELI), are cutting speed as $140 \mathrm{rpm}$, Nose radius $1.2 \mathrm{~mm}$, Feed $0.051 \mathrm{~mm} / \mathrm{rev}$ and depth of cut is $0.5 \mathrm{~mm}$.

- ANOVA test revels the R-Sq value of model as $88.74 \%$

- Pareto chart and ANOVA table of GRG, indicate cutting speed and feed are significant parameters followed by cutting speed and depth of cut.

- The average error while comparing calculated GRG and Regression GRG was 7.63\%

- The GRG values predicted by ANN model were having average error of $3.75 \%$

\section{References}

Akahori, T., \& Niinomi, M. (1998). Fracture characteristics of fatigued Ti-6Al-4V ELI as an implant material. Materials Science and Engineering: A, 243(1-2), 237-243.

Anand, G., Alagumurthi, N., Elansezhian, R., Palanikumar, K., \& Venkateshwaran, N. (2018). Investigation of drilling parameters on hybrid polymer composites using grey relational analysis, regression, fuzzy logic, and ANN models. Journal of the Brazilian Society of Mechanical Sciences and Engineering, 40(4).

Asiltürk, I., \& Çunkaş, M. (2011). Modeling and prediction of surface roughness in turning operations using artificial neural network and multiple regression method. Expert Systems with Applications, $38(5), 5826-5832$.

Ganta, V., Sagar, K. S., \& Chakradhar, D. (2017). Multi objective optimisation of thermally enhanced machining parameters of Inconel 718 using grey relational analysis. International Journal of Machining and Machinability of Materials, 19(1), 57-75.

Che-Haron, C. H., \& Jawaid, A. (2005). The effect of machining on surface integrity of titanium alloy Ti-6\% Al-4\% v. Journal of Materials Processing Technology, 166(2), 188-192.

Gosai, M., \& Bhavsar, S. N. (2016). Experimental Study on Temperature Measurement in Turning Operation of Hardened Steel (EN36). Procedia Technology, 23, 311-318.

Lee, H. S., Yoon, J. H., Park, C. H., Ko, Y. G., Shin, D. H., \& Lee, C. S. (2007). A study on diffusion bonding of superplastic Ti-6Al-4V ELI grade. Journal of Materials Processing Technology, 187-188, 526-529.

Maiyar, L. M., Ramanujam, R., Venkatesan, K., \& Jerald, J. (2013). Optimization of machining parameters for end milling of Inconel 718 super alloy using Taguchi based grey relational analysis. Procedia Engineering, 64, 1276-1282.

Moura, R. R., da Silva, M. B., Machado, ??lisson R., \& Sales, W. F. (2015). The effect of application of cutting fluid with solid lubricant in suspension during cutting of Ti-6Al-4V alloy. Wear, 332-333, 762-771.

Nalbant, M., Gökkaya, H., Toktaş, I., \& Sur, G. (2009). The experimental investigation of the effects of 
uncoated, PVD- and CVD-coated cemented carbide inserts and cutting parameters on surface roughness in $\mathrm{CNC}$ turning and its prediction using artificial neural networks. Robotics and ComputerIntegrated Manufacturing, 25(1), 211-223.

Narutaki, N., Murakoshi, A., Motonishi, S., \& Takeyama, H. (1983). Study on Machining of Titanium Alloys. CIRP Annals - Manufacturing Technology, 32(1), 65-69.

Nath, C., Kapoor, S. G., \& Srivastava, A. K. (2017). Finish turning of Ti-6Al-4V with the atomizationbased cutting fluid (ACF) spray system. Journal of Manufacturing Processes, 28, 464-471.

Niinomi, M. (1998). Mechanical properties of biomedical titanium alloys. Materials Science and Engineering: A, 243(1-2), 231-236.

Prasad, Y. V. R. K., Seshacharyulu, T., Medeiros, S. C., \& Frazier, W. G. (2001). Influence of oxygen content on the forging response of equiaxed $(\alpha+\beta)$ preform of Ti-6Al-4V: Commercial vs. ELI grade. Journal of Materials Processing Technology, 108(3), 320-327.

Ren, J., Zhou, J., \& Wei, J. (2015). Optimization of cutter geometric parameters in end milling of titanium alloy using the grey-taguchi method. Advances in Mechanical Engineering, 7(2).

Sarıkaya, M., \& Güllü, A. (2015). Multi-response optimization of minimum quantity lubrication parameters using Taguchi-based grey relational analysis in turning of difficult-to-cut alloy Haynes 25. Journal of Cleaner Production, 91, 347-357.

Sulaiman, M.A., Che Haron, C.H. , Ghani, J.A., \& Kasim, M. S. (2013). Optimization of Turning Parameters for Titanium Alloy Ti-6Al-4V ELI Using the Response Surface Method (RSM). Journal of Advanced Manufacturing Technology, 7(2), 11-28.

Tamilselvi, S., Raman, V., \& Rajendran, N. (2006). Corrosion behaviour of Ti-6Al-7Nb and Ti-6Al-4V ELI alloys in the simulated body fluid solution by electrochemical impedance spectroscopy. Electrochimica Acta, 52(3), 839-846.

Valera, H. Y., \& Bhavsar, S. N. (2014). Experimental investigation of surface roughness and power consumption in turning operation of EN 31 Alloy steel. Procedia Technology, 14, 528-534.

Vinayagamoorthy, R., \& Anthony Xavior, M. (2014). Parametric optimization on multi-objective precision turning using grey relational analysis. Procedia Engineering, 97, 299-307.

Xie, J., Luo, M. J., Wu, K. K., Yang, L. F., \& Li, D. H. (2013). Experimental study on cutting temperature and cutting force in dry turning of titanium alloy using a non-coated micro-grooved tool. International Journal of Machine Tools and Manufacture, 73, 25-36.

Yildiz, T., Irez, A. B., \& Sur, G. (2016, July). Effect of cementite carbide tool coating type and tool radius on cutting performance. In Mechanical and Aerospace Engineering (ICMAE), 2016 7th International Conference on (pp. 78-82). IEEE.

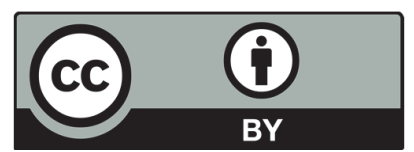

(C) 2019 by the authors; licensee Growing Science, Canada. This is an open access article distributed under the terms and conditions of the Creative Commons Attribution (CCBY) license (http://creativecommons.org/licenses/by/4.0/). 